\title{
The prognostic value of preoperative neutrophils, platelets, lymphocytes, monocytes and calculated ratios in patients with laryngeal squamous cell cancer
}

\author{
Chiyao Hsueh ${ }^{1,2}$, Lei Tao ${ }^{1,2}$, Ming Zhang ${ }^{1,2}$, Wenjun $\mathrm{CaO}^{3}$, Hongli Gong ${ }^{1,2}$, Jian Zhou ${ }^{1,2}$ \\ and Liang Zhou ${ }^{1,2}$ \\ ${ }^{1}$ Department of Otolaryngology, Eye Ear Nose \& Throat Hospital, Fudan University, Shanghai, PR China \\ 2 Shanghai Key Clinical Disciplines of Otorhinolaryngology, Shanghai, PR China \\ ${ }^{3}$ Department of Clinical Laboratory, Eye \& ENT Hospital, Shanghai Medical College, Fudan University, PR China \\ Correspondence to: Liang Zhou, email: zhoulent@126.com \\ Keywords: lymphocytes, neutrophil-to-lymphocyte ratio, lymphocyte-to-monocyte ratio, laryngeal squamous carcinoma, prog- \\ nosis \\ Received: December 27, 2016 Accepted: February 20, $2017 \quad$ Published: March 15, 2017
}

Copyright: Hsueh et al. This is an open-access article distributed under the terms of the Creative Commons Attribution License 3.0 (CC BY 3.0), which permits unrestricted use, distribution, and reproduction in any medium, provided the original author and source are credited.

\section{ABSTRACT}

This study was aimed to examine the prognostic value of preoperative neutrophils, platelets, lymphocytes, monocytes and calculated ratios in patients with laryngeal squamous cell cancer (LSCC). From January 2007 to December 2011, 979 patients with LSCC were enrolled in our study. Preoperative neutrophils, platelets, lymphocytes, monocytes, neutrophil-to-lymphocyte ratio (NLR), platelet-to-lymphocyte ratio (PLR), and lymphocyte-to-monocyte ratio (LMR) were analyzed. Besides well-established clinicopathological prognostic factors, we evaluated the independent prognostic relevance of these hematological parameters by Cox regression models in disease-free survival (DFS) and cancer-specific survival (CSS). We found patients in the highest tertile of NLR (>2.40), PLR (>111.00) were at significantly higher risk of DFS and CSS $(P<0.05)$ compared with those in the lowest tertile after multivariate analysis, whereas presenting significantly higher risk in the lowest tertile of lymphocytes $\left(<1.60 \times 10^{\circ} / \mathrm{L}\right)$ and LMR $(<3.50)$. Additionally, the tertile category of NLR as well as PLR increased and lymphocytes as well as LMR decreased in shorter DFS and CSS by the Kaplan-Meier method and the log-rank test. In conclusion, this study indicated that preoperative lymphocytes, NLR, PLR and LMR were significantly associated with LSCC progression, DFS and CSS, and these hematological parameters could be considered independent prognostic values for patients with LSCC.

\section{INTRODUCTION}

Laryngeal cancer is one of the most common head and neck cancers that remains as the remarkable cause of morbidity and mortality. The predominant histological type of laryngeal cancer is laryngeal squamous cell cancer (LSCC). The estimated incidence and mortality of laryngeal cancer were 26,400 and 14,500 cases in China in 2015 [1]. Recently, conventional treatment options in LSCC have mainly included surgery, radiotherapy, chemotherapy either used alone or in combination [2], and the clinical classification system of the Union
International Contre le Cancer (UICC) and the American Joint Committee on cancer (AJCC) were frequently and universally used for the appropriate selections of surgical procedure and prognostic evaluation. Despite improvements in diagnostic approach and treatment, the previously identified trend in 5-year relative survival rates indicated that the clinical outcome for LSCC does not have significantly improved in the past thirty years (from 59.6\% to $66.8 \%$ ) [3]. Moreover, it is inadequate that the current TNM staging system has been based almost exclusively on the anatomic extent of LSCC to identify patients with LSCC at high-risk recurrence and prognosis [4]. Hence, 
it is of great significance to find out more parameters that can provide more efficient and useful information of prognosis after treatment.

Since Virchow first observed the close connection between inflammation and malignancy when he found the presence of leukocytes in neoplastic tissue in 1863, increasing evidence has shown that inflammation participates in components of tumorigenesis and tumor progression [5,6]. A series of peripheral inflammatory cells such as neutrophil, platelet, lymphocyte and monocyte, have been performed prognostic value in various cancers. Huang, SH. et al. [7] reported high circulating neutrophil count and monocyte count predicted the inferior overall survival (OS) and recurrence-free survival (RFS). Wang, H. et al. [8] reported a higher level of platelet in patients with pancreatic cancer correlated with poorer tumor progression, faster metastasis and lower OS. In addition, Rachidi, S. et al. [9] reported higher lymphocyte count in patients in LSCC was associated with longer OS. Therefore, the variation of these inflammatory cells, including neutrophilic leukocytosis, thrombocytosis, monocytosis and lymphopenia could supply prognostic value in patients with cancer.
Recently, several studies have clarified those parameters of preoperative neutrophil-to-lymphocyte ratio (NLR), platelet-to-lymphocyte ratio (PLR) and lymphocyte-to-monocyte ratio (LMR) were closely related to predict prognosis in patients with various cancers such as head and neck squamous cell cancer (HNSCC) [9-13], hepatocellular carcinoma [14] and metastatic renal cell carcinoma [15]. In addition, compared with other numerous prognostic factors, the advantages of inflammation-based prognostic parameters are simple, widely available, and inexpensive from the preoperative evaluation of the blood test. However, to our knowledge, few studies correlated with the association of clinical significance with respect to the prognostic value of these preoperative inflammatory cells remained uncertain in patients with LSCC [16-18]. Moreover, the sample size of some of these studies was limited. Therefore, the aim of this study was to evaluate the effect of neutrophils, platelets, lymphocytes, monocytes, NLR, PLR and LMR on the prognosis of preoperative patients with LSCC.
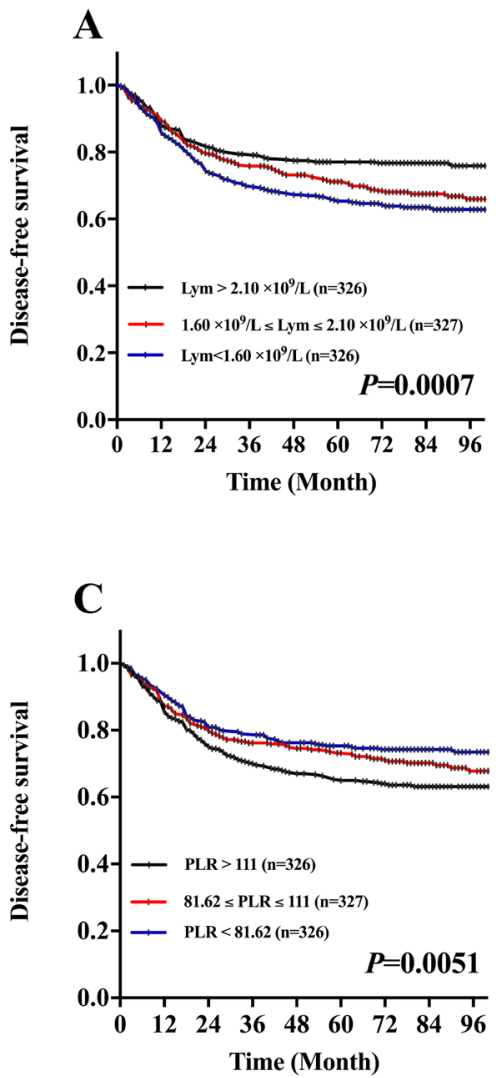
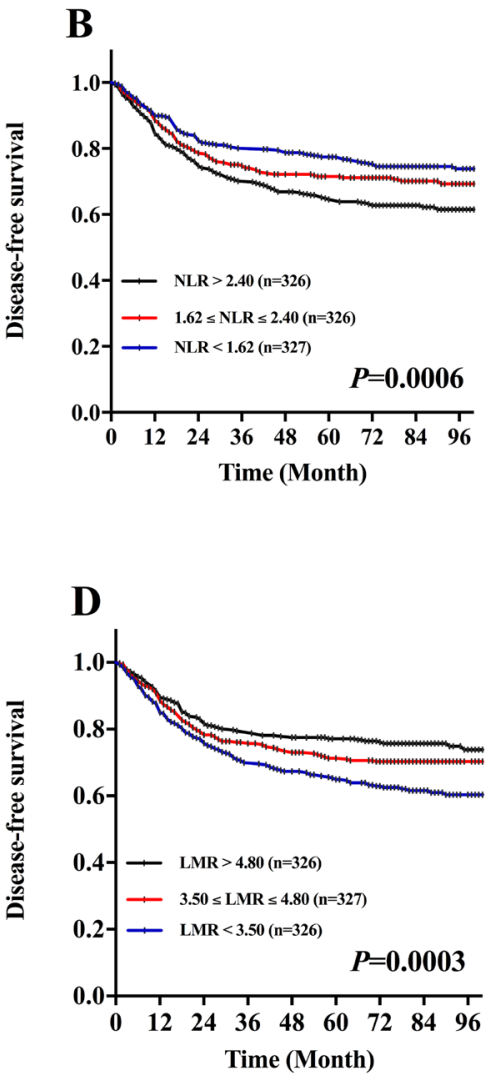

Figure 1: Kaplan-Meier DFS curves stratified by the tertile distribution in terms of lymphocytes (Lym), neutrophil-tolymphocyte ratio (NLR), platelet-to-lymphocyte ratio (PLR) and lymphocyte-to-monocyte ratio (LMR). A. DFS curves stratified based on lymphocyte count category. B. DFS curves stratified based on NLR category. C. DFS curves stratified based on PLR category. D .DFS curves stratified based on LMR category. 
Table 1: Clinicopathological demographics and clinical characteristics in patients (n=979) with LSCC.

\begin{tabular}{|c|c|}
\hline Characteristics & Number of patients $(\%)$ \\
\hline Age, years $(<60 / \geq 60)$ & $469(47.9 \%) / 510(52.1 \%)$ \\
\hline Gender (Male / Female) & $955(97.5 \%) / 24(2.5 \%)$ \\
\hline Smoking history (No / Yes) & $313(32.8 \%) / 666(67.2 \%)$ \\
\hline Drinking history (No / Yes) & $610(62.3 \%) / 369(37.7 \%)$ \\
\hline Tumor subsite (Supraglottic / Glottic / Subglottic) & $226(23.1 \%) / 741(75.7 \%) / 12(1.2 \%)$ \\
\hline Tumor size $(\leq 2 \mathrm{~cm} />2 \mathrm{~cm})$ & $631(64.5 \%) / 348(35.5 \%)$ \\
\hline \multicolumn{2}{|l|}{ Tumor stage } \\
\hline $\mathrm{T} 1 / \mathrm{T} 2$ & $232(23.7 \%) / 387(39.5 \%)$ \\
\hline $\mathrm{T} 3 / \mathrm{T} 4$ & $297(30.3 \%) / 63(6.4 \%)$ \\
\hline \multicolumn{2}{|l|}{ Lymph node stage } \\
\hline No & $866(88.5 \%)$ \\
\hline $\mathrm{N} 1 / \mathrm{N} 2 / \mathrm{N} 3$ & $36(3.7 \%) / 67(6.8 \%) / 10(1.0 \%)$ \\
\hline \multicolumn{2}{|l|}{ Clinical stage ${ }^{*}$} \\
\hline I / I & $232(23.7 \%) / 356(36.4 \%)$ \\
\hline III / IV & $263(26.8 \%) / 128(13.1 \%)$ \\
\hline \multicolumn{2}{|l|}{ Differentiation grade } \\
\hline Well \& moderate / Poor & $809(82.6 \%) / 30(3.1 \%)$ \\
\hline Unknown & $140(14.3 \%)$ \\
\hline \multicolumn{2}{|l|}{ Operation therapy } \\
\hline Total laryngectomy & $408(41.7 \%)$ \\
\hline Vertical / Frontolateral partial laryngectomy & $350(35.8 \%)$ \\
\hline CHP \& CHEP & $120(12.3 \%)$ \\
\hline Cordectomy ( $\mathrm{CO}_{2}$ laser $)$ & $76(7.7 \%)$ \\
\hline Horizontal supraglottic partial laryngectomy & $25(2.5 \%)$ \\
\hline
\end{tabular}

* According to the $7^{\text {th }}$ American Joint Committee on cancer (AJCC) stage system.

\section{RESULTS}

\section{Patients demographics and clinical characteristics}

Our study included 979 patients with LSCC underwent the laryngectomy. The median observation period (from the date of surgery to the last follow-up) for the entire study population was 79 month (range: 3 to 116 months; mean $\pm \mathrm{SD}=73.14 \pm 30.25$ months). The 5-year follow-up rate was $72.5 \%$. Patients demographics and clinical characteristics are shown in Table 1. The clinical data included 955 males $(97.5 \%)$ and 24 females $(2.5 \%)$ with a mean age of $60.81 \pm 9.68$ years (range 27 to 89 years). The 666 patients $(67.2 \%)$ had smoking history, and $369(32.8 \%)$ patients had drinking history. The site of the primary tumor was divided into the glottic cancer $(n=741$, $75.7 \%)$, supraglottic cancer $(n=226,23.1 \%)$ and subglottic cancer $(n=12,1.2 \%)$. The 348 patients $(35.5 \%)$ had the diameter of LSCC $(>2 \mathrm{~cm})$ confirmed by pathology. The 113 patients $(11.5 \%)$ had lymph node metastasis. The 588 patients $(60.1 \%)$ and 391 patients $(39.9 \%)$ had the early stage and advanced stage with LSCC. The surgical therapies which all patients underwent were divided into partial laryngectomy $(571,58.3 \%)$ and total laryngectomy (408, 41.7\%). 
Table 2: The mean hematological value of neutrophils $\left(\times 10^{9} / \mathrm{L}\right)$, platelets $\left(\times 10^{9} / \mathrm{L}\right)$, lymphocytes $\left(\times 10^{9} / \mathrm{L}\right)$, monocytes $\left(\times 10^{9} / \mathrm{L}\right)$ by patient's demographic and clinical characteristics.

\begin{tabular}{|c|c|c|c|c|c|c|c|c|}
\hline & $\begin{array}{c}\text { Neu } \\
(\text { mean } \pm \text { SD) }\end{array}$ & $P$ & $\begin{array}{c}\text { Plt } \\
(\text { mean } \pm \text { SD })\end{array}$ & $P$ & $\begin{array}{c}\text { Lym } \\
(\operatorname{mean} \pm \text { SD) }\end{array}$ & $P$ & $\begin{array}{c}\text { Mon } \\
(\operatorname{mean} \pm \text { SD) }\end{array}$ & $P$ \\
\hline Age (years) & & 0.438 & & $<0.001$ & & $<0.001$ & & 0.179 \\
\hline$<60$ & $3.92 \pm 1.40$ & & $192.22 \pm 55.25$ & & $2.08 \pm 0.68$ & & $0.49 \pm 0.17$ & \\
\hline$\geq 60$ & $3.85 \pm 1.35$ & & $175.08 \pm 53.04$ & & $1.80 \pm 0.59$ & & $0.48 \pm 0.17$ & \\
\hline Gender & & 0.002 & & 0.783 & & 0.048 & & 0.007 \\
\hline Male & $3.90 \pm 1.37$ & & $183.37 \pm 54.87$ & & $1.94 \pm 0.65$ & & $0.49 \pm 0.17$ & \\
\hline Female & $3.03 \pm 1.29$ & & $180.25 \pm 50.94$ & & $1.68 \pm 0.53$ & & $0.39 \pm 0.15$ & \\
\hline Smoking history & & 0.994 & & 0.059 & & 0.014 & & 0.020 \\
\hline No & $3.88 \pm 1.52$ & & $178.48 \pm 51.55$ & & $1.86 \pm 0.65$ & & $0.46 \pm 0.17$ & \\
\hline Yes & $3.88 \pm 1.30$ & & $185.55 \pm 56.10$ & & $1.97 \pm 0.65$ & & $0.49 \pm 0.18$ & \\
\hline Drinking history & & 0.982 & & 0.350 & & 0.118 & & 0.021 \\
\hline No & $3.88 \pm 1.41$ & & $182.02 \pm 53.79$ & & $1.91 \pm 0.64$ & & $0.47 \pm 0.17$ & \\
\hline Yes & $3.88 \pm 1.31$ & & $185.40 \pm 56.34$ & & $1.98 \pm 0.66$ & & $0.50 \pm 0.17$ & \\
\hline Tumor subsite & & 0.006 & & 0.029 & & 0.056 & & $<0.001$ \\
\hline Supraglottic & $4.12 \pm 1.51$ & & $191.21 \pm 64.58$ & & $1.86 \pm 0.61$ & & $0.52 \pm 0.18$ & \\
\hline Glottic \& Subglottic & $3.81 \pm 1.32$ & & $180.91 \pm 51.26$ & & $1.96 \pm 0.66$ & & $0.47 \pm 0.17$ & \\
\hline Tumor size & & $<0.001$ & & $<0.001$ & & 0.026 & & $<0.001$ \\
\hline$\leq 2 \mathrm{~cm}$ & $3.69 \pm 1.27$ & & $178.24 \pm 50.25$ & & $1.97 \pm 0.66$ & & $0.46 \pm 0.16$ & \\
\hline$>2 \mathrm{~cm}$ & $4.23 \pm 1.48$ & & $192.44 \pm 61.12$ & & $1.87 \pm 0.63$ & & $0.51 \pm 0.19$ & \\
\hline Tumor stage & & $<0.001 *$ & & $<0.001^{*}$ & & $0.002^{*}$ & & $<0.001^{*}$ \\
\hline $\mathrm{T} 1$ & $3.73 \pm 1.24$ & & $176.87 \pm 49.13$ & & $2.00 \pm 0.69$ & & $0.46 \pm 0.16$ & \\
\hline $\mathrm{T} 2$ & $3.74 \pm 1.28$ & & $178.28 \pm 50.52$ & & $1.96 \pm 0.67$ & & $0.48 \pm 0.16$ & \\
\hline T3 & $4.00 \pm 1.46$ & & $188.30 \pm 60.01$ & & $1.91 \pm 0.59$ & & $0.49 \pm 0.17$ & \\
\hline $\mathrm{T} 4$ & $4.77 \pm 1.37$ & & $214.08 \pm 61.87$ & & $1.67 \pm 0.54$ & & $0.58 \pm 0.25$ & \\
\hline Lymph node stage & & 0.017 & & 0.724 & & 0.280 & & 0.044 \\
\hline No & $3.84 \pm 1.34$ & & $183.07 \pm 53.10$ & & $1.94 \pm 0.65$ & & $0.48 \pm 0.17$ & \\
\hline $\mathrm{N}+$ & $4.21 \pm 1.55$ & & $185.00 \pm 66.37$ & & $1.87 \pm 0.67$ & & $0.51 \pm 0.18$ & \\
\hline Clinical stage & & $<0.001^{* *}$ & & $0.001^{* *}$ & & $0.012^{* * * *}$ & & $<0.001^{* * *}$ \\
\hline I & $3.73 \pm 1.24$ & & $176.87 \pm 49.13$ & & $2.00 \pm 0.69$ & & $0.46 \pm 0.16$ & \\
\hline II & $3.71 \pm 1.25$ & & $180.10 \pm 50.81$ & & $1.96 \pm 0.66$ & & $0.47 \pm 0.16$ & \\
\hline III & $3.97 \pm 1.43$ & & $185.63 \pm 57.09$ & & $1.91 \pm 0.61$ & & $0.48 \pm 0.16$ & \\
\hline IV & $4.46 \pm 1.62$ & & $199.00 \pm 66.30$ & & $1.78 \pm 0.61$ & & $0.55 \pm 0.23$ & \\
\hline Differentiation grade & & 0.398 & & 0.776 & & 0.244 & & 0.863 \\
\hline Well \& moderate & $3.93 \pm 1.38$ & & $184.87 \pm 55.49$ & & $1.92 \pm 0.64$ & & $0.49 \pm 0.18$ & \\
\hline Poor & $4.41 \pm 1.55$ & & $181.93 \pm 52.75$ & & $2.06 \pm 0.65$ & & $0.48 \pm 0.15$ & \\
\hline
\end{tabular}

Bold values represent that $P$ were two-sided and $P<0.05$ was statistically significant.

Abbreviations: Neu, neutrophils $\left(\times 10^{9} / \mathrm{L}\right)$; Plt, platelets $\left(\times 10^{9} / \mathrm{L}\right)$; Lym, lymphocytes $\left(\times 10^{9} / \mathrm{L}\right)$; Mon, monocytes $\left(\times 10^{9} / \mathrm{L}\right)$. ${ }^{*} P<0.05$ for the significant difference between the mean hematological parameters of T1, T2 and T3 categories and of T4 categories, respectively (1-way ANOVA with the Bonferroni post hoc test).

${ }^{* *} P<0.05$ for the significant difference between the mean hematological parameters of I, II and III stages and of IV stages, respectively (1-way ANOVA with the Bonferroni post hoc test).

${ }^{* * *} P<0.05$ for the significant difference between the mean hematological parameters of I and II stages and of III stages, respectively (1-way ANOVA with the Bonferroni post hoc test).

\section{Hematological parameters related to patient characteristics}

The mean hematological absolute value of neutrophil, platelet, lymphocyte and monocyte counts (Table 2), and these mean hematological parameters of 
Table 3: The mean hematological value of neutrophil-to-lymphocyte ratio (NLR), platelet-to-lymphocyte ratio (PLR), and lymphocyte-to-monocyte ratio (LMR) by patient's demographic and clinical characteristics.

\begin{tabular}{|c|c|c|c|c|c|c|}
\hline & $\begin{array}{c}\text { NLR } \\
(\operatorname{mean} \pm \mathbf{S D})\end{array}$ & $P$ & $\begin{array}{c}\text { PLR } \\
(\text { mean } \pm \text { SD })\end{array}$ & $P$ & $\begin{array}{c}\text { LMR } \\
(\text { mean } \pm \text { SD })\end{array}$ & $\boldsymbol{P}$ \\
\hline Age (years) & & $<0.001$ & & 0.035 & & 0.001 \\
\hline$<60$ & $2.07 \pm 1.04$ & & $100.30 \pm 38.69$ & & $4.58 \pm 1.70$ & \\
\hline$\geq 60$ & $2.39 \pm 1.34$ & & $105.96 \pm 44.64$ & & $4.18 \pm 2.14$ & \\
\hline Gender & & 0.119 & & 0.283 & & 0.471 \\
\hline Male & $2.24 \pm 1.23$ & & $103.02 \pm 42.19$ & & $4.36 \pm 1.96$ & \\
\hline Female & $1.85 \pm 0.75$ & & $112.33 \pm 31.20$ & & $4.65 \pm 1.56$ & \\
\hline Smoking history & & 0.057 & & 0.178 & & 0.944 \\
\hline No & $2.35 \pm 1.41$ & & $105.88 \pm 44.61$ & & $4.36 \pm 1.75$ & \\
\hline Yes & $2.18 \pm 1.11$ & & $102.01 \pm 40.65$ & & $4.37 \pm 2.04$ & \\
\hline Drinking history & & 0.279 & & 0.419 & & 0.286 \\
\hline No & $2.27 \pm 1.26$ & & $104.09 \pm 42.69$ & & $4.42 \pm 2.09$ & \\
\hline Yes & $2.18 \pm 1.15$ & & $101.85 \pm 40.78$ & & $4.28 \pm 1.69$ & \\
\hline Tumor subsite & & 0.005 & & 0.005 & & $<0.001$ \\
\hline Supraglottic & $2.43 \pm 1.25$ & & $110.53 \pm 44.79$ & & $3.87 \pm 1.47$ & \\
\hline Glottic \& Subglottic & $2.17 \pm 1.20$ & & $101.06 \pm 40.86$ & & $4.52 \pm 2.05$ & \\
\hline Tumor size & & $<0.001$ & & $<0.001$ & & $<0.001$ \\
\hline$\leq 2 \mathrm{~cm}$ & $2.07 \pm 1.08$ & & $98.60 \pm 39.20$ & & $4.58 \pm 2.08$ & \\
\hline$>2 \mathrm{~cm}$ & $2.53 \pm 1.40$ & & $111.67 \pm 45.43$ & & $3.99 \pm 1.63$ & \\
\hline Tumor stage & & $<0.001 *$ & & $<0.001 *$ & & $<0.001^{*}$ \\
\hline $\mathrm{T} 1$ & $2.07 \pm 1.06$ & & $95.84 \pm 35.33$ & & $4.67 \pm 1.75$ & \\
\hline $\mathrm{T} 2$ & $2.14 \pm 1.16$ & & $100.34 \pm 42.96$ & & $4.48 \pm 2.29$ & \\
\hline $\mathrm{T} 3$ & $2.28 \pm 1.11$ & & $105.13 \pm 38.23$ & & $4.23 \pm 1.60$ & \\
\hline $\mathrm{T} 4$ & $3.23 \pm 1.96$ & & $139.52 \pm 55.29$ & & $3.25 \pm 1.41$ & \\
\hline Lymph node stage & & 0.015 & & 0.334 & & 0.014 \\
\hline No & $2.20 \pm 1.19$ & & $102.78 \pm 41.68$ & & $4.42 \pm 2.00$ & \\
\hline $\mathrm{N}+$ & $2.50 \pm 1.38$ & & $106.83 \pm 44.17$ & & $3.95 \pm 1.50$ & \\
\hline Clinical stage & & $<0.001^{* *}$ & & $<0.001^{* *}$ & & $<0.001^{* *}$ \\
\hline I & $2.07 \pm 1.06$ & & $95.84 \pm 35.33$ & & $4.67 \pm 1.75$ & \\
\hline II & $2.10 \pm 1.08$ & & $100.60 \pm 42.06$ & & $4.53 \pm 2.33$ & \\
\hline III & $2.27 \pm 1.10$ & & $104.57 \pm 40.36$ & & $4.26 \pm 1.62$ & \\
\hline IV & $2.84 \pm 1.77$ & & $121.30 \pm 50.36$ & & $3.62 \pm 1.54$ & \\
\hline Differentiation grade & & 0.773 & & 0.236 & & 0.561 \\
\hline Well \& moderate & $2.27 \pm 1.23$ & & $104.40 \pm 41.45$ & & $4.30 \pm 1.97$ & \\
\hline Poor & $2.20 \pm 1.17$ & & $95.28 \pm 38.72$ & & $4.51 \pm 1.52$ & \\
\hline
\end{tabular}

Bold values represent that $P$ were two-sided and $P<0.05$ was statistically significant.

Abbreviations: NLR, neutrophil-to-lymphocyte ratio; PLR, platelet-to-lymphocyte ratio; LMR, lymphocyte-to-monocyte ratio; $\mathrm{SD}$, standard deviation.

${ }^{*} P<0.05$ for the significant difference between the mean hematological parameters of T1, T2 and T3 stages and of T4 stages, respectively (1-way ANOVA with the Bonferroni post hoc test).

${ }^{* *} P<0.05$ for the significant difference between the mean hematological parameters of I, II and III stages and of IV stages, respectively (1-way ANOVA with the Bonferroni post hoc test). 
Table 4: Univariate Cox proportional hazards regression analysis for disease-free survival (DFS) and cancer-specific survival (CSS) in patients with laryngeal squamous cell cancer (LSCC).

\begin{tabular}{|c|c|c|c|c|}
\hline \multirow{2}{*}{ Characteristics } & DFS & \multirow{2}{*}{$\boldsymbol{P}$} & CSS & \multirow{2}{*}{$\boldsymbol{P}$} \\
\hline & $\operatorname{HR}(95 \% \mathrm{CI})$ & & HR $(95 \%$ CI $)$ & \\
\hline Age (years) & & 0.135 & & 0.007 \\
\hline$<60$ & 1.000 & & 1.000 & \\
\hline$\geq 60$ & $1.191(0.947-1.499)$ & & $1.465(1.111-1.932)$ & \\
\hline Gender & & 0.076 & & 0.125 \\
\hline Female & 1.000 & & 1.000 & \\
\hline Male & $2.800(0.898-8.733)$ & & $2.975(0.739-11.976)$ & \\
\hline Smoking history & & 0.153 & & 0.760 \\
\hline NO & 1.000 & & 1.000 & \\
\hline YES & $1.202(0.934-1.546)$ & & $1.047(0.780-1.405)$ & \\
\hline Drinking history & & 0.113 & & 0.297 \\
\hline NO & 1.000 & & 1.000 & \\
\hline YES & $1.206(0.957-1.519)$ & & $1.158(0.879-1.524)$ & \\
\hline Tumor subsite & & $<0.001$ & & $<0.001$ \\
\hline Glottic \& Subglottic & 1.000 & & 1.000 & \\
\hline Supraglottic & $2.196(1.730-2.789)$ & & $2.215(1.672-2.935)$ & \\
\hline Differentiation grade & & 0.014 & & 0.234 \\
\hline Well \& moderate & 1.000 & & 1.000 & \\
\hline Poor & $1.878(1.133-3.110)$ & & $1.471(0.779-2.780)$ & \\
\hline Tumor stage & & $<0.001$ & & $<0.001$ \\
\hline $\mathrm{T} 1-\mathrm{T} 2$ & 1.000 & & 1.000 & \\
\hline T3-T4 & $2.794(2.220-3.516)$ & & $2.737(2.081-3.599)$ & \\
\hline Lymph node stage & & $<0.001$ & & $<0.001$ \\
\hline N0 & 1.000 & & 1.000 & \\
\hline $\mathrm{N}+$ & $3.052(2.316-4.022)$ & & $2.880(2.086-3.976)$ & \\
\hline Neutrophils $\left(\times 10^{9} / \mathrm{L}\right)$ & & 0.248 & & 0.567 \\
\hline$<3.20$ & 1.000 & & 1.000 & \\
\hline $3.20-4.30$ & $1.154(0.868-1.535)$ & & $1.079(0.770-1.513)$ & \\
\hline$>4.30$ & $1.271(0.959-1.685)$ & & $1.196(0.858-1.668)$ & \\
\hline Platelets $\left(\times 10^{9} / \mathrm{L}\right)$ & & 0.149 & & 0.642 \\
\hline$<157.00$ & 1.000 & & 1.000 & \\
\hline $157.00-200.00$ & $0.829(0.623-1.104)$ & & $0.883(0.631-1.235)$ & \\
\hline$>200.00$ & $1.096(0.836-1.437)$ & & $1.028(0.742-1.426)$ & \\
\hline Lymphocytes $\left(\times 10^{9} / \mathrm{L}\right)$ & & 0.003 & & $<0.001$ \\
\hline$>2.10$ & 1.000 & & 1.000 & \\
\hline $1.60-2.10$ & $1.403(1.043-1.888)$ & 0.025 & $1.896(1.307-2.752)$ & 0.001 \\
\hline$<1.60$ & $1.646(1.232-2.200)$ & 0.001 & $2.197(1.525-3.164)$ & $<0.001$ \\
\hline Monocytes $\left(\times 10^{9} / \mathrm{L}\right)$ & & 0.474 & & 0.816 \\
\hline$<0.40$ & 1.000 & & 1.000 & \\
\hline $0.40-0.50$ & $1.033(0.777-1.373)$ & & $1.060(0.759-1.482)$ & \\
\hline$>0.50$ & $1.176(0.891-1.553)$ & & $1.114(0.799-1.554)$ & \\
\hline NLR & & 0.003 & & 0.003 \\
\hline$<1.62$ & 1.000 & & 1.000 & \\
\hline $1.62-2.40$ & $1.223(0.909-1.645)$ & 0.184 & $1.517(1.061-2.169)$ & 0.022 \\
\hline$>2.40$ & $1.618(1.221-2.145)$ & 0.001 & $1.836(1.297-2.598)$ & 0.001 \\
\hline PLR & & 0.021 & & 0.009 \\
\hline
\end{tabular}




\begin{tabular}{lcccc}
$<81.62$ & 1.000 & & 1.000 & \\
$81.62-111.00$ & $1.207(0.901-1.619)$ & 0.208 & $1.350(0.946-1.925)$ & 0.097 \\
$>111.00$ & $1.488(1.122-1.972)$ & $\mathbf{0 . 0 0 6}$ & $1.708(1.214-2.402)$ & $\mathbf{0 . 0 0 2}$ \\
$\begin{array}{l}\text { LMR } \\
>4.80\end{array}$ & & $\mathbf{0 . 0 0 1}$ & & $<\mathbf{0 . 0 0 1}$ \\
$3.50-4.80$ & 1.000 & & 1.000 & \\
$<3.50$ & $1.223(0.907-1.650)$ & 0.187 & $1.321(0.916-1.906)$ & 0.137 \\
\hline
\end{tabular}

Bold values represent that $\mathrm{P}$ were two-sided and $P<0.05$ was statistically significant.

Abbreviation: DFS, disease-free survival; CSS, cancer-specific disease HR: hazard ratio; CI, confidence interval; NLR, neutrophil-to-lymphocyte ratio; PLR, platelet-to-lymphocyte ratio; LMR, lymphocyte-to-monocyte ratio.

NLR, PLR, LMR (Table 3) compared with age, gender, smoking history, drinking history, tumor subsite, tumor size, local and regional extension category of the primary tumor, clinical stage and differentiation grade.

In the clinical demographic, the significantly increased number of platelets, lymphocytes, LMR were discovered in the patients under 60 years old, whereas the decreased number of NLR and PLR presented in our study. Moreover, female patients had significantly lower counts of neutrophil, lymphocyte and monocyte than male patients. Patients with smoking history had higher counts of lymphocyte and monocyte than those of non-smoking patients. Only monocytes had a significant difference in the drinking history. However, NLR, PLR, and LMR had no significant variation in gender, smoking history and drinking history groups.

In the clinicopathological characteristics, we found the number of neutrophils, platelets, monocytes, NLR and PLR increased in clinical stage, but lymphocytes and LMR decreased. All the hematological parameters do have significant variation in the tumor stage and size. Neutrophils, monocytes, NLR and LMR significantly correlated with the regional extension category of LSCC. Moreover, apart from the lymphocytes, others had a variation between supraglottic and glottic \& subglottic. Differentiation grade, however, had no significant variation in our study.

Supplementary Table 1, online only, indicated neutrophils, platelets, lymphocytes, monocytes, NLR, PLR and LMR were based on the tertile distribution to examine the correlation with clinical parameters.

\section{Univariate and Multivariate Analysis of Prognostic factors}

The result of the univariate Cox proportional hazards $(\mathrm{CPH})$ regression model for clinicopathologic parameters and these hematological categories defined by the tertile distribution of disease-free survival (DFS) and cancer-specific survival (CSS) was shown in Table 4. In univariate analysis, the independent variables were age $(<60$ vs $\geq 60)$, gender, smoking history, drinking history, tumor subsite, differentiation grade, local (T1-
$\mathrm{T} 2$ vs T3-T4) and regional (N0 vs $\mathrm{N}+$ ) extension as well as neutrophils, platelets, lymphocytes, monocytes, NLR, PLR and LMR categories, respectively, as defined by the tertile distribution.

We considered tumor subsite, the local and regional extension category, and lymphocytes, NLR, PLR and LMR categories defined by the tertile were significant independent factors of DFS and CSS. As a result, patients with NLR in the highest tertile $(>2.40)$ had lower DFS (HR: $1.618,95 \%$ CI: $1.221-2.145, P=0.001$ ) and CSS (HR: $1.836,95 \%$ CI: $1.297-2.598, P=0.001$ ) than those in the lowest tertile $(<1.62)$, as well as lower CSS in the middle tertile $(1.62-2.40$, HR: $1.517,95 \%$ CI: 1.061 2.169, $P=0.022)$. Patients with PLR in the highest tertile $(>111.00)$ had lower DFS (HR: $1.488,95 \% \mathrm{CI}$ : 1.122-1.972, $P=0.006$ ) and CSS (HR: $1.708,95 \% \mathrm{CI}$ : $1.214-2.402, P=0.002)$ than those in the lowest tertile $(<81.62)$. Conversely, patients with lymphocytes in the lowest tertile $\left(<1.60 \times 10^{9} / \mathrm{L}\right)$ had lower DFS (HR: 1.646, 95\% CI: 1.232-2.200, $P=0.001$ ) and CSS (HR: 2.197, $95 \%$ CI: $1.525-3.164, p<0.001)$ than those in the highest tertile $\left(>2.10 \times 10^{9} / \mathrm{L}\right)$ as well as lower DFS (HR: 1.403 , 95\% CI: $1.043-1.888, P=0.025)$ and CSS (HR: 1.896 , 95\% CI: $1.307-2.752, P=0.001)$ in the middle tertile $\left(1.60-2.10 \times 10^{9} / \mathrm{L}\right)$. Additionally, Patients with LMR in the lowest tertile $(<3.50)$ had lower DFS (HR: $1.671,95 \%$ CI: $1.260-2.217, P<0.001)$ and CSS (HR: $1.975,95 \% \mathrm{CI}$ : $1.403-2.779, P<0.001)$ than those in the highest tertile $(>4.80)$.

Patient's demographics and clinical characteristics for prognostic values of DFS and CSS were further investigated by multivariate $\mathrm{CPH}$ regression model. These hematological categories with $p<0.05$ in univariate $\mathrm{CPH}$ regression model were included in multivariate $\mathrm{CPH}$ regression model in Table 5.

In multivariate $\mathrm{CPH}$ regression model for DFS, we found tumor stage $(p<0.001)$, lymph node stage $(p<0.001)$, lymphocytes in the lowest tertile $(P=0.001)$, NLR in the highest tertile $(P=0.017)$, PLR in the highest tertile $(P=0.039)$ and LMR in the lowest tertile $(P=0.024)$ were independent prognostic factors.

In multivariate $\mathrm{CPH}$ regression model for CSS, the tumor stage $(P<0.001)$, lymph node stage $(P=0.003)$, lymphocytes in the lowest tertile $(P<0.001)$ and the 
Table 5: Multivariate Cox proportional hazards regression for disease-free survival (DFS) and cancer-specific survival (CSS) in patients with laryngeal squamous cell cancer (LSCC).

\begin{tabular}{|c|c|c|c|c|}
\hline \multirow[b]{2}{*}{ Characteristics } & DFS & \multirow{2}{*}{$\boldsymbol{P}$} & CSS & \multirow{2}{*}{$\boldsymbol{P}$} \\
\hline & HR $(95 \%$ CI) & & HR $(95 \%$ CI) & \\
\hline Tumor stage & & $<0.001$ & & $<0.001$ \\
\hline $\mathrm{T} 1-\mathrm{T} 2$ & 1.000 & & 1.000 & \\
\hline T3-T4 & $1.982(1.538-2.553)$ & & $2.236(1.664-3.004)$ & \\
\hline Lymph node stage & & $<0.001$ & & 0.003 \\
\hline No & 1.000 & & 1.000 & \\
\hline $\mathrm{N}+$ & $1.970(1.431-2.712)$ & & $1.766(1.210-2.577)$ & \\
\hline Lymphocytes $\left(\times 10^{9} / \mathrm{L}\right)$ & & 0.004 & & 0.001 \\
\hline$>2.10$ & 1.000 & & 1.000 & \\
\hline $1.60-2.10$ & $1.289(0.947-1.754)$ & 0.106 & $1.718(1.182-2.498)$ & 0.005 \\
\hline$<1.60$ & $1.656(1.227-2.235)$ & 0.001 & $1.977(1.365-2.863)$ & $<\mathbf{0 . 0 0 1}$ \\
\hline NLR & & 0.037 & & 0.106 \\
\hline$<1.62$ & 1.000 & & 1.000 & \\
\hline $1.62-2.4$ & $1.104(0.810-1.506)$ & 0.531 & $1.289(0.899-1.849)$ & 0.167 \\
\hline$>2.40$ & $1.432(1.066-1.925)$ & 0.017 & $1.464(1.029-2.084)$ & 0.034 \\
\hline PLR & & 0.111 & & 0.064 \\
\hline$<81.62$ & 1.000 & & 1.000 & \\
\hline $81.62-111.00$ & $1.146(0.844-1.555)$ & 0.384 & $1.219(0.854-1.740)$ & 0.276 \\
\hline$>111.00$ & $1.360(1.015-1.821)$ & 0.039 & $1.502(1.065-2.118)$ & 0.020 \\
\hline LMR & & 0.025 & & 0.022 \\
\hline$>4.80$ & 1.000 & & 1.000 & \\
\hline $3.50-4.80$ & $1.017(0.744-1.390)$ & 0.915 & $1.119(0.772-1.622)$ & 0.553 \\
\hline$<3.50$ & $1.407(1.046-1.891)$ & 0.024 & $1.565(1.101-2.227)$ & 0.013 \\
\hline
\end{tabular}

Bold values represent that $P$ were two-sided and $P<0.05$ was statistically significant. Abbreviation: DFS, disease-free survival; CSS, cancer-specific disease HR: hazard ratio; CI, confidence interval; NLR, neutrophil-to-lymphocyte ratio; PLR, platelet-to-lymphocyte ratio; LMR, lymphocyte-to-monocyte ratio.

middle tertile $(P=0.005)$, NLR in the highest tertile $(P=0.034)$, PLR in the highest tertile $(P=0.020)$ and LMR in the lowest tertile $(P=0.013)$ were independent prognostic factors.

As a result, our study found that the tumor stage, lymph node stage, lymphocytes, NLR, PLR and LMR were independent prognostic factors in DFS and CSS of LSCC.

\section{Disease-free survival (DFS) and cancer-specific survival (CSS) outcome}

The estimated 5-year DFS of the 979 patients was $71.16 \%$ (range: $1-116$ months; median \pm SD: $75 \pm 35.99$ months) in our study. During the followed-up period, a total of 296 patients $(30.2 \%)$ with LSCC recurrence, including 42 patients (14.2\%) had local LSCC recurrence, 88 patients $(29.7 \%)$ had regional LSCC recurrence, 63 patients $(21.3 \%)$ had locoregional LSCC recurrence, and 103 patients $(34.8 \%)$ had distant metastasis.

To evaluate the association of DFS and these hematological categories including NLR, PLR, lymphocyte and LMR, Kaplan-Meier survival analysis and log-rank tests were performed that the 5-year DFS in patients with NLR categories were $64.5 \%$ (the highest tertile), $71.5 \%$ (the middle tertile), and $77.4 \%$ (the lowest tertile), respectively $(P=0.0006$, Figure $1 \mathrm{~B})$. In the perspective of PLR categories were $65.0 \%$ (the highest tertile), $73.2 \%$ (the middle tertile), and $75.3 \%$ (the lowest tertile), respectively ( $P=0.0051$, Figure $1 C)$. Conversely, the 5-year DFS in the patients with lymphocyte categories were $77.1 \%$ (the highest tertile), $71.2 \%$ (the middle tertile), and $65.3 \%$ (the lowest tertile), respectively ( $P=0.0007$, Figure 1A). In the perspective of LMR categories were $77.2 \%$ (the highest tertile), $71.3 \%$ (the middle tertile), and $65.0 \%$ (the lowest tertile), respectively ( $P=0.0003$, Figure 1D). 
The estimated 5-year CSS of the 979 patients was $80.76 \%$ (range: $3-116$ months; median \pm SD: $79 \pm 30.28$ months) and overall survival was $79.46 \%$ (range: 3-116 months; median \pm SD: $79 \pm 35.99$ months). A total of 209 patients $(21.3 \%)$ died because of tumor progression in our study.

To evaluate the association of CSS and these hematological categories including NLR, PLR, lymphocyte and LMR, Kaplan-Meier survival analysis and log-rank tests were performed that the 5-year CSS in patients with NLR categories were $75.2 \%$ (the highest tertile), $79.4 \%$ (the middle tertile), and $87.7 \%$ (the lowest tertile), respectively $(P=0.0005$, Figure 2B). In the perspective of PLR categories were $75.5 \%$ (the highest tertile), $81.8 \%$ (the middle tertile), and $84.9 \%$ (the lowest tertile), respectively ( $P=0.0018$, Figure $2 \mathrm{C}$ ). Conversely, the 5-year CSS in patients with lymphocyte categories were $88.2 \%$ (the highest tertile), $79.7 \%$ (the middle tertile), and $74.4 \%$ (the lowest tertile), respectively $(P<0.0001$, Figure 2A). In the perspective of LMR categories were $86.5 \%$ (highest tertile), $81.3 \%$ (middle tertile), and $74.5 \%$ (lowest tertile), respectively $(P<0.0001$, Figure 2D).
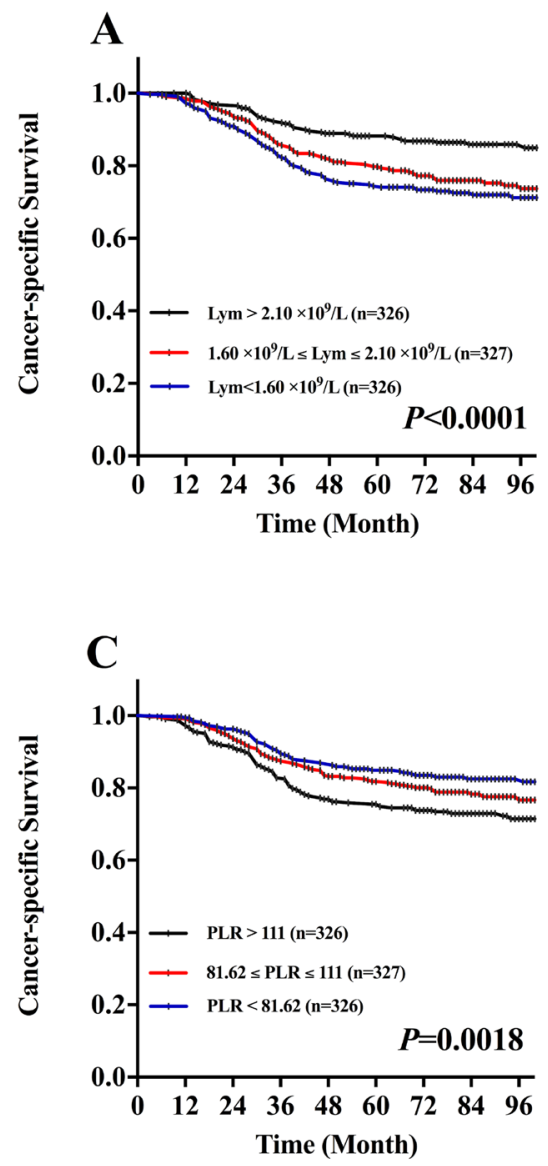

Additionally, this study indicated these inflammatory parameters were based on the tertile distribution to examine the correlation with patients of clinical stage IIIIV. In DFS, these parameters had no significant relation in patients of stage II-IV (Figure S1). Whereas in CSS, we found that lymphocytes, NLR and LMR have significant variation in patients of stage III-IV (Figure S2).

\section{DISCUSSION}

This study evaluated the preoperative prognostic value of the comprehensive inflammatory absolute counts (neutrophils, platelets, lymphocytes and monocytes) and calculated ratios (NLR, PLR and LMR) for patients with LSCC. Our study demonstrated that preoperative lower neutrophils, monocytes, platelets, NLR, PLR and higher lymphocytes as well as LMR correlated to LSCC progression, including tumor size, tumor stage and clinical stage. Furthermore, our result first showed that the absolute count of the peripheral lymphocyte was the most significantly related to DFS and CSS.
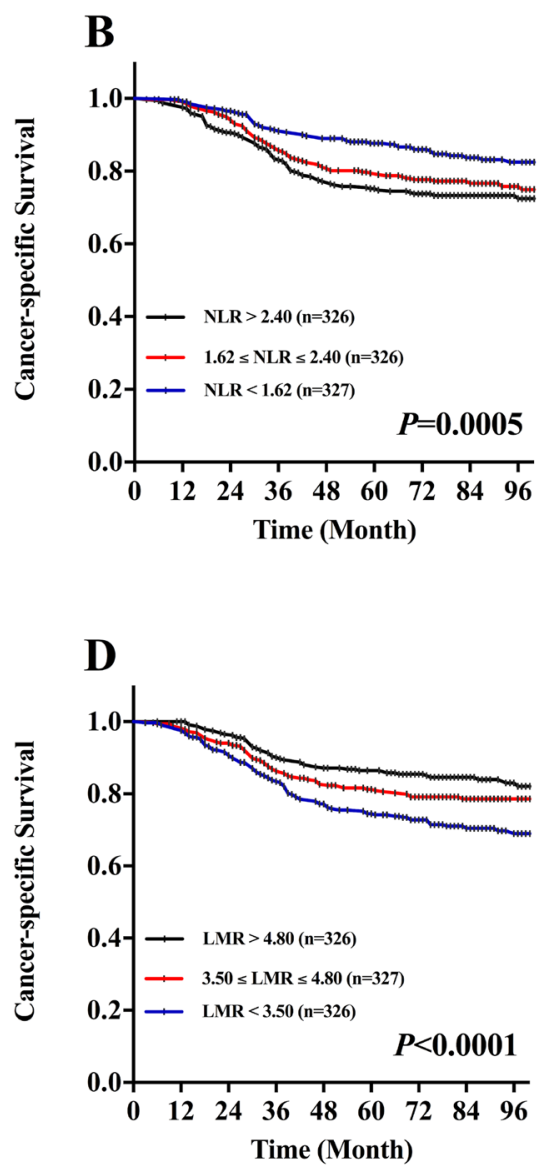

Figure 2: Kaplan-Meier CSS curves stratified by the tertile distribution in terms of lymphocytes (Lym), neutrophil-tolymphocyte ratio (NLR), platelet-to-lymphocyte ratio (PLR) and lymphocyte-to-monocyte ratio (LMR). A. CSS curves stratified based on lymphocyte count category. B. CSS curves stratified based on NLR category. C. CSS curves stratified based on PLR category. D. CSS curves stratified based on LMR category. 
In the evaluation of the relationship between these mean hematological absolute counts and patient's demographics and characteristics, our results matched with those of other authors in head and neck cancers. This study found several increases in the preoperative absolute counts of neutrophil [7, 10, 19], platelet [20] and monocyte [7, $10]$, and a decrease in the preoperative absolute count of lymphocyte [21] in significant correlation to advanced LSCC. However, Kara, M. et al. [22] and Duzlu, M. et al. [23] didn't find the association between these hematological absolute values and the LSCC-related variables. We found that the male patients had higher counts of neutrophils, lymphocytes and monocytes compared with the female patients, as other authors observed [7, 10, 19]. In addition, patients with the history of smoking and drinking presented significant increases in the pretreatment counts of lymphocyte and monocyte, as Valero, C. et al. [10] reported. In order to eliminate the mutual influence between these mean hematological absolute counts and patient's demographics, we evaluated the relationship between these hematological parameters (NLR, PLR and LMR) and the LSCC-related variables. We found that these mean hematological calculated ratios (NLR, PLR and LMR) were significantly related to LSCC progression as other observed in NLR and PLR. [16, 17, $22,24]$. We found trends in the value of these different inflammatory parameters and calculated ratios based on the LSCC-related variables of the patients. These trends could play a valuable role in the LSCC progression.
In multivariate $\mathrm{CPH}$ regression model for DFS and CSS, we found that lymphocytes, NLR, PLR and LMR were independent prognostic factors. Most studies demonstrated that neutrophil infiltration can produce and secrete angiogenesis-regulating growth factors, cytokines and protease to promote tumor cell growth and cause poorer survival ratio [25-27]. Moreover, neutrophils have been demonstrated to suppress the cytotoxic activity of lymphocytes, natural killer cells, and activated $\mathrm{T}$ cells through the production of reactive oxygen species, arginase, and nitric oxide [28, 29].An increasing in platelet number and activity can not only interact with cancer cells to facilitate tumor metastases through direct contact and secreted bioactive proteins, but also release mediators of both tumor angiogenesis and osteoclast resorption to promote tumor cell growth and survival [30-32]. Peripheral monocytes leave the bloodstream and migrate into tissues where, following conditioning by local growth factors, both microbial products and pro-inflammatory cytokines can differentiate into tissue macrophages and dendritic cells populations [33]. In addition, many studies reported that these cells can produce and secrete various cytokines to promote tumorigenesis, cancer progression and metastasis [27, 34-36].

Although the prognostic values of NLR, PLR and LMR depended mainly on the number of neutrophils, platelets and monocytes compared with lymphocytes, the prognostic values of neutrophils, platelets and monocytes were more limited than those of lymphocytes.

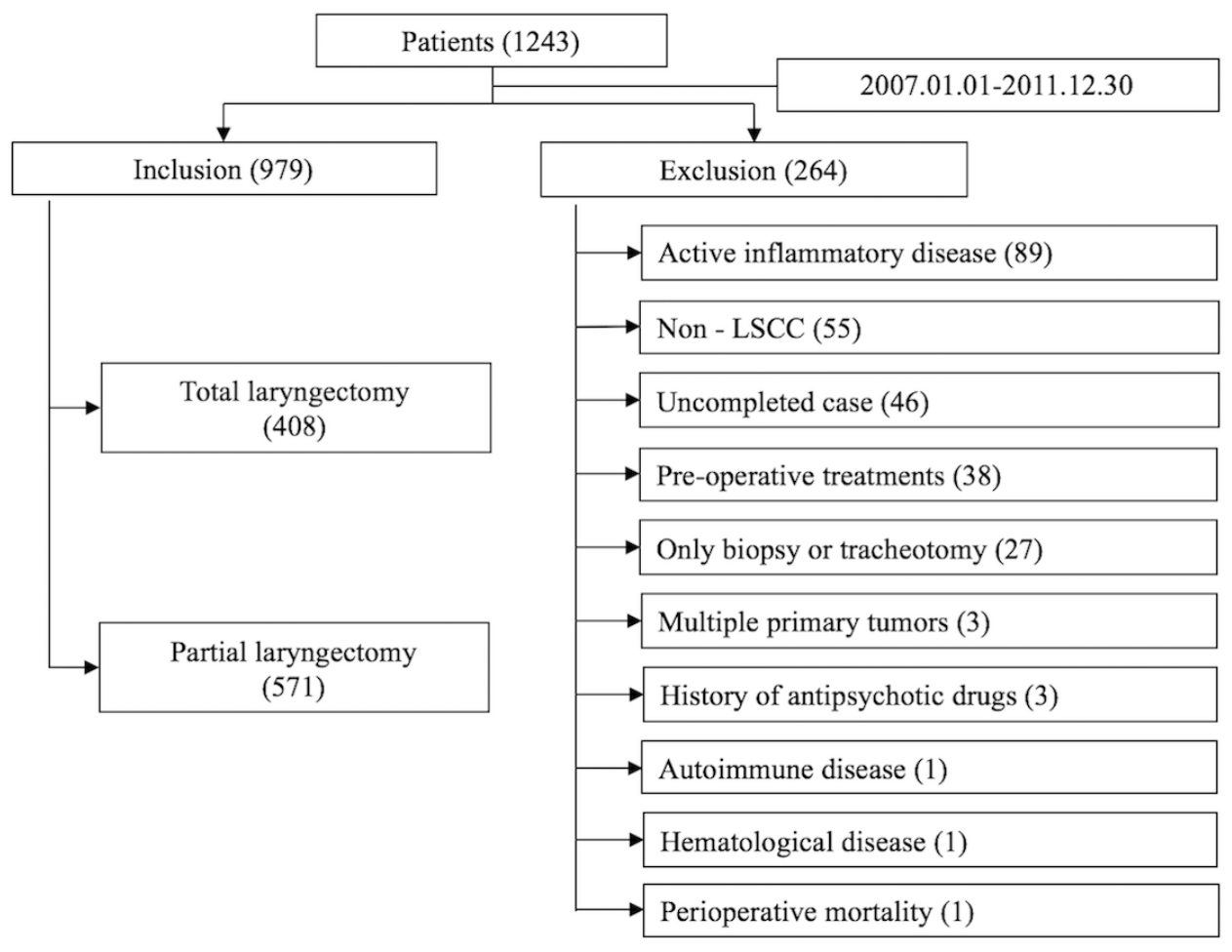

Figure 3: Patient selection process. 
Lymphocytes are very important hematological cells of the host immune system that are robust for activation of an effective antitumor response, and the varying inflammatory responses in LSCC are possibly proved to be instrumental in prognosis [37, 38]. Furthermore, decreased numbers of lymphocytes in LSCC was generally deemed to present the state of immunosuppression, and may attenuate lymphocyte-mediated antitumor immunity [39].

When the DFS and CSS curves were analyzed by the Kaplan-Meier method and the difference was assessed by the log-rank test, we found that the lymphocytes, NLR, PLR and LMR were all associated with the prognosis of patients with LSCC in terms of both DFS and CSS. Although all patients enrolled in this study were treated with partial or total laryngectomy, the patients with lymphocytes in the lowest tertile $(<1.60)$, NLR in the highest tertile $(>2.40)$, PLR in the highest tertile $(>111.00)$ and LMR in the lowest tertile $(<3.50)$ were associated with poorer DFS. In LSCC, previous studies evaluating the prognostic value of NLR found the relationship between increased NLR and the deterioration in DFS, CSS and OS[16-18, 24], whereas the value of NLR lacked prognostic DFS in the study of Wong, B. et al. [16]. Only one study demonstrated the relationship between increased PLR and the deterioration in CSS [22]. In head and neck cancers, many studies found the number of lymphocytes positively correlate with OS and CSS $[9,10]$. Sun, W. et al. [40] found the pretreatment PLR $(\geq 167.2)$ was significantly associated with shorter progression-free survival (PFS) in patients with nasopharyngeal carcinoma. In addition, Kano, S. et al. [11] found the pretreatment LMR $(<3.22)$ was a significantly associated with shorter DFS and OS in patients with head and neck cancer. Therefore, the lymphocytes, NLR, PLR and LMR could be the independent prognostic marker in patients with LSCC.

However, there are several limitations in this study. This was a single-center retrospective study based on 979 eligible patients with LSCC before the laryngectomy. Furthermore, the association between peripheral hematological parameters and treatment outcome for laryngectomy remains uncertain. Therefore, further studies are needed to illuminate the mechanism between these inflammatory parameters and prognosis in patients with LSCC.

In conclusion, this study indicated that preoperative lymphocytes, NLR, PLR and LMR were significantly associated with cancer progression, disease-free survival and cancer-specific survival, and these hematological parameters could be considered independent prognostic values for patients with LSCC.

\section{MATERIALS AND METHODS}

\section{Study population}

A clinical data was collected using a primary cohort of the consecutive patients undergoing partial or total laryngectomy as the first curative treatment option for LSCC at the Eye \& ENT Hospital of Fudan University between January $1^{\text {th }}, 2007$ to $31^{\text {th }}$, December 2011. The inclusion criteria for this study were as follows: (1) primary laryngeal squamous cell cancer confirmed by pathology and classified by the $7^{\text {th }}$ edition of the TNM-UICC/AJCC stage classification; (2) signed the informed consent before operation; (3) the blood samples were collected before the operation and (4) complete clinical, laboratory, imaging, and follow-up data. The exclusion criteria were as follows: (1) cancer of uncertain origin or probable metastatic LSCC by CT or MRI scans; (2) mixed type of primary LSCC confirmed histopathologically; (3) pre-operative treatments such as radiotherapy or chemotherapy; (4) active inflammatory disease or concomitant infection; (5) autoimmune disease or treatment with steroids [9]; (6) hematological disorders or treatment within one year before operation; (7) antipsychotic drugs history and (8) perioperative mortality. The 1243 patients met the inclusion and exclusion criterion, among which 264 patients were later excluded from this study, so that the final data of 979 patients were obtained and analyzed in this study (Figure 3). Ethic approval was obtained from the Ethical Committees of the Eye \& ENT Hospital (Shanghai, China). All patients were followed-up by telephone, message and outpatient records for every 3 months with the content of survival status, disease progress and time of death during first 2 years, and every 6 months thereafter until death. The last followup was 30 September, 2016. Disease-free survival (DFS) was recorded from the date of laryngectomy to the data of recurrence within the fellow-up period. Cancer-specific survival (CSS) was recorded from the date of surgery until death because of the intercurrent disease within the fellowup period. Overall survival (OS) was recorded from the date of surgery until death.

\section{Data collection}

We reviewed the patient files for the clinical, histopathological, and laboratory data. The following preoperative hematological parameters were collected in ethylenediaminetetraacetic acid-containing tubes within 2 weeks before laryngectomy treatment. Preoperative complete blood counts included neutrophil count, platelet count, lymphocyte count and monocyte count were measured with the Mindary BC-5500 (Shenzhen, China) automatic blood counting system. The NLR, PLR and 
LMR were calculated by the division of the absolute values of the corresponding hematological parameters.

\section{Statistical analysis}

Patient demographics (gender, age, smoking status and drinking status) and clinical characteristics (tumor subsite, tumor size, local and regional extension category of the primary tumor, clinical stage, differentiation grade, Surgical therapy and neck dissection) were displayed as frequency counts and percentages. All statistical analyses were performed with SPSS version 22.0 software (Chicago, IL, USA). The descriptive data were presented as mean \pm standard deviation. We classified neutrophil, platelet, lymphocyte, and monocyte counts, NLR, PLR and LMR into 3 categories based on the tertile distribution in the study population. The t-test and 1-way analysis of variance (ANOVA) were taken account of the assessment between these mean hematological absolute values and patient's demographics \& characteristics. The upper tertile included the highest count, followed by the middle tertile and finally the lower tertile categorized the patients with the lowest count. The neutrophils were divided into $<3.20$ neutrophils $\times 10^{9} / \mathrm{L}$ (the lowest tertile), 3.20 to 4.30 neutrophils $\times 10^{9} / \mathrm{L}$ (the middle tertile) and $>4.30 \times 10^{9} / \mathrm{L}$ (the highest tertile). The platelets were divided into $<$ 157.00 platelets $\times 10^{9} / \mathrm{L}$ (the lowest tertile), 157.00 to 200.00 platelets $\times 10^{\%} / \mathrm{L}$ (the middle tertile) and $>200$ platelets $\times 10^{9} / \mathrm{L}$ (the highest tertile). The lymphocytes were divided into $<1.60$ lymphocytes $\times 10^{9} / \mathrm{L}$ (the lowest tertile), 1.60 to 2.10 lymphocytes $\times 10^{9} / \mathrm{L}$ (the middle tertile) and $>2.10$ lymphocytes $\times 10^{9} / \mathrm{L}$ (the highest tertile). The monocytes were divided into $<0.40$ monocytes $\times 10^{9} / \mathrm{L}$ (the lowest tertile), 0.40 to 0.50 monocytes $\times 10^{9} / \mathrm{L}$ (the middle tertile) and $>0.50$ monocytes $\times 10^{9} / \mathrm{L}$ (the highest tertile). The NLR were divided into NLR $<1.62$ (the lowest tertile), 1.62 to 2.40 (the middle tertile) and $>2.40$ (the highest tertile). The PLR were divided into PLR $<81.62$ (the lowest tertile), 81.62 to 111.00 (the middle tertile) and $>111.00$ (the highest tertile). The LMR were divided into LMR $<3.50$ (the lowest tertile), 3.50 to 4.80 (the middle tertile) and $>4.80$ (the highest tertile). The chi-square test $\left({ }^{2}\right)$ was used to analyze the relationship between clinicopathologic parameters and these hematological categories defined by the tertile distribution. Univariate analysis was performed with the $\mathrm{CPH}$ regression model to test independent clinicopathologic parameters and these hematological categories defined by the tertile distribution, and these hematological categories with $P<0.05$ were included in DFS and CSS curves before the application of the multivariate $\mathrm{CPH}$ regression model. As the hematological parameters were with significance $(P<0.05)$ in the use of multivariate $\mathrm{CPH}$ regression model, the regression model DFS and CSS curves were plotted using the Kaplan-Meier method respectively, and the difference was assessed by the log-rank test. Multivariate analysis was performed with the Cox regression model to test independent significance while adjusting for covariates.; data are presented as hazard ratios (HR) and 95\% confidence intervals $(95 \% \mathrm{CI})$. All $P$ presented were two-tailed and $P<0.05$ was considered significant.

\section{Abbreviations}

LSCC=laryngeal squamous cell cancer, $\mathrm{NLR}=$ neutrophil-to-lymphocyte ratio, $\mathrm{PLR}=$ platelet-tolymphocyte ratio, $\mathrm{LMR}=$ lymphocyte-to-monocyte ratio, $\mathrm{DFS}=$ disease-free survival; $\mathrm{CSS}=$ cancer-specific survival, $\mathrm{OS}=$ overall survival, $\mathrm{HR}=$ hazard ratio, $\mathrm{CI}=$ confidence interval.

\section{ACKNOWLEDGMENTS}

This study was supported by the Science and Technology Commission of Shanghai Municipality, China [Grant Nos. 12J1402100 and 16411950101], Hospital Development Center [Grant No. SHDC12015114].

\section{Author contributions}

Chiyao, Hsueh, Liang, Zhou, Hongli, Gong, Jian, Zhou, Wenjun, Cao conceived the study, participated in drafting the final manuscript. Chiyao, Hsueh, Liang, Zhou, Lei, Tao, Ming, Zhang analyzed the data and completed the final draft of the manuscript. Chiyao, Hsueh prepared all the figures. All authors reviewed the manuscript.

\section{CONFLICTS OF INTEREST}

None declared.

\section{Additional information}

Competing financial interests: The authors declare no competing financial interests. The sponsor or funding organization had no role in the design or conduct of this research.

\section{REFERENCES}

1. Chen W, Zheng R, Baade PD, Zhang S, Zeng H, Bray F, Jemal A, Yu XQ, He J. Cancer statistics in China, 2015. CA Cancer J Clin. 2016; 66:115-32. https://doi.org/10.3322/ caac. 21338

2. Chawla S, Carney AS. Organ preservation surgery for laryngeal cancer. Head Neck Oncol. 2009; 1:12. https://doi. org/10.1186/1758-3284-1-12

3. Howlader N, Noone A, Krapcho M, Miller D, Bishop K, Altekruse S, Kosary C, Yu M, Ruhl J, Tatalovich Z, 
Mariotto A. SEER Cancer Statistics Review, 1975-2013, National Cancer Institute.

4. Takes RP, Rinaldo A, Silver CE, Piccirillo JF, Haigentz M Jr, Suárez C, Van der Poorten V, Hermans R, Rodrigo JP, Devaney KO, Ferlito A. Future of the TNM classification and staging system in head and neck cancer. Head Neck. 2010; 32:1693-711. https://doi.org/10.1002/hed.21361

5. Balkwill F, Mantovani A. Inflammation and cancer: back to Virchow? Lancet. 2001; 357:539-45. https://doi. org/10.1016/S0140-6736(00)04046-0

6. Coussens LM, Werb Z. Inflammation and cancer. Nature. 2002; 420:860-67. https://doi.org/10.1038/nature01322

7. Huang SH, Waldron JN, Milosevic M, Shen X, Ringash J, Su J, Tong L, Perez-Ordonez B, Weinreb I, Bayley AJ, Kim J, Hope A, Cho BC, et al. Prognostic value of pretreatment circulating neutrophils, monocytes, and lymphocytes in oropharyngeal cancer stratified by human papillomavirus status. Cancer. 2015; 121:545-55. https://doi.org/10.1002/ cncr. 29100

8. Wang H, Gao J, Bai M, Liu R, Li H, Deng T, Zhou L, Han R, Ge S, Huang D, Ba Y. The pretreatment platelet and plasma fibrinogen level correlate with tumor progression and metastasis in patients with pancreatic cancer. Platelets. 2014; 25:382-87. https://doi.org/10.3109/09537104.2013.8 27782

9. Rachidi S, Wallace K, Wrangle JM, Day TA, Alberg AJ, Li Z. Neutrophil-to-lymphocyte ratio and overall survival in all sites of head and neck squamous cell carcinoma. Head Neck. 2016 (Suppl 1); 38:E1068-74. https://doi. org/10.1002/hed.24159

10. Valero C, Pardo L, López M, García J, Camacho M, Quer M, León X. Pretreatment count of peripheral neutrophils, monocytes, and lymphocytes as independent prognostic factor in patients with head and neck cancer. Head Neck. 2017; 39:219-26. https://doi.org/10.1002/hed.24561

11 Kano S, Homma A, Hatakeyama H, Mizumachi T, Sakashita T, Kakizaki T, Fukuda S. Pretreatment lymphocyte-tomonocyte ratio as an independent prognostic factor for head and neck cancer. Head Neck. 2017; 39:247-53. https://doi. org/10.1002/hed.24576

12. Rassouli A, Saliba J, Castano R, Hier M, Zeitouni AG. Systemic inflammatory markers as independent prognosticators of head and neck squamous cell carcinoma. Head Neck. 2015; 37:103-10. https://doi.org/10.1002/ hed.23567

13. Kawakita D, Tada Y, Imanishi Y, Beppu S, Tsukahara K, Kano S, Ozawa H, Okami K, Sato Y, Shimizu A, Sato Y, Fushimi C, Takase S, et al. Impact of hematological inflammatory markers on clinical outcome in patients with salivary duct carcinoma: a multi-institutional study in Japan. Oncotarget. 2017; 8:1083-91. https://doi.org/10.18632/ oncotarget. 13565

14. Qi X, Li J, Deng H, Li H, Su C, Guo X. Neutrophilto-lymphocyte ratio for the prognostic assessment of hepatocellular carcinoma: A systematic review and metaanalysis of observational studies. Oncotarget. 2016; 7:45283-301. https://doi.org/10.18632/oncotarget.9942

15. Gu L, Ma X, Wang L, Li H, Chen L, Li X, Zhang Y, Xie Y, Zhang X. Prognostic value of a systemic inflammatory response index in metastatic renal cell carcinoma and construction of a predictive model. Oncotarget. Oncotarget. 2017; 8:52094-103. https://doi.org/10.18632/ oncotarget.10626

16. Wong BY, Stafford ND, Green VL, Greenman J. Prognostic value of the neutrophil-to-lymphocyte ratio in patients with laryngeal squamous cell carcinoma. Head Neck. 2016 (Suppl 1); 38:E1903-08. https://doi.org/10.1002/hed.24346

17. Fu Y, Liu W, OuYang D, Yang A, Zhang Q. Preoperative Neutrophil-to-lymphocyte Ratio Predicts Long-term Survival in Patients Undergoing Total Laryngectomy With Advanced Laryngeal Squamous Cell Carcinoma: A Singlecenter Retrospective Study. Medicine (Baltimore). 2016; 95:e2689. https://doi.org/10.1097/MD.0000000000002689

18. Tu XP, Qiu QH, Chen LS, Luo XN, Lu ZM, Zhang SY, Chen SH. Preoperative neutrophil-to-lymphocyte ratio is an independent prognostic marker in patients with laryngeal squamous cell carcinoma. BMC Cancer. 2015; 15:743. https://doi.org/10.1186/s12885-015-1727-6

19. He JR, Shen GP, Ren ZF, Qin H, Cui C, Zhang Y, Zeng YX, Jia WH. Pretreatment levels of peripheral neutrophils and lymphocytes as independent prognostic factors in patients with nasopharyngeal carcinoma. Head Neck. 2012; 34:1769-76. https://doi.org/10.1002/hed.22008

20. Pardo L, Valero C, López M, García J, Camacho M, Quer M, León X. The prognostic value of pretreatment platelet count in patients with head and neck squamous cell carcinoma. Auris Nasus Larynx. 2017; 44:313-18. https:// doi.org/10.1016/j.anl.2016.06.009

21. Cho O, Oh YT, Chun M, Noh OK, Hoe JS, Kim H. Minimum absolute lymphocyte count during radiotherapy as a new prognostic factor for nasopharyngeal cancer. Head Neck. 2016 (Suppl 1); 38:E1061-67. https://doi. org/10.1002/hed.24158

22. Kara M, Uysal S, Altinişik U, Cevizci S, Güçlü O, Dereköy FS. The pre-treatment neutrophil-to-lymphocyte ratio, platelet-to-lymphocyte ratio, and red cell distribution width predict prognosis in patients with laryngeal carcinoma. Eur Arch Otorhinolaryngol. 2017; 274:535-42. https://doi. org/10.1007/s00405-016-4250-8

23. Duzlu M, Karamert R, Tutar H, Karaloglu F, Sahin M, Cevizci R. Neutrophil-lymphocyte ratio findings and larynx carcinoma: a preliminary study in Turkey. Asian Pac J Cancer Prev. 2015; 16:351-54. https://doi.org/10.7314/ APJCP.2015.16.1.351

24. Zeng YC, Chi F, Xing R, Xue M, Wu LN, Tang MY, Wu R. Pre-treatment neutrophil-to-lymphocyte ratio predicts prognosis in patients with locoregionally advanced laryngeal carcinoma treated with chemoradiotherapy. Jpn J Clin Oncol. 2016; 46:126-31. https://doi.org/10.1093/jjco/ 
hyv 175

25. Gastardelo TS, Cunha BR, Raposo LS, Maniglia JV, Cury PM, Lisoni FC, Tajara EH, Oliani SM. Inflammation and cancer: role of annexin A1 and FPR2/ALX in proliferation and metastasis in human laryngeal squamous cell carcinoma. PLoS One. 2014; 9:e111317. https://doi. org/10.1371/journal.pone.0111317

26. Benevides L, da Fonseca DM, Donate PB, Tiezzi DG, De Carvalho DD, de Andrade JM, Martins GA, Silva JS. IL17 Promotes Mammary Tumor Progression by Changing the Behavior of Tumor Cells and Eliciting Tumorigenic Neutrophils Recruitment. Cancer Res. 2015; 75:3788-99. https://doi.org/10.1158/0008-5472.CAN-15-0054

27. Galdiero MR, Bonavita E, Barajon I, Garlanda C, Mantovani A, Jaillon S. Tumor associated macrophages and neutrophils in cancer. Immunobiology. 2013; 218:1402-10. https://doi.org/10.1016/j.imbio.2013.06.003

28. Müller I, Munder $M$, Kropf $P$, Hänsch GM. Polymorphonuclear neutrophils and T lymphocytes: strange bedfellows or brothers in arms? Trends Immunol. 2009; 30:522-30. https://doi.org/10.1016/j.it.2009.07.007

29. Pillay J, Kamp VM, van Hoffen E, Visser T, Tak T, Lammers JW, Ulfman LH, Leenen LP, Pickkers P, Koenderman L. A subset of neutrophils in human systemic inflammation inhibits $\mathrm{T}$ cell responses through Mac-1. J Clin Invest. 2012; 122:327-36. https://doi.org/10.1172/ JCI57990

30. Sharma D, Brummel-Ziedins KE, Bouchard BA, Holmes CE. Platelets in tumor progression: a host factor that offers multiple potential targets in the treatment of cancer. J Cell Physiol. 2014; 229:1005-15. https://doi.org/10.1002/ jcp.24539

31. Naugler WE, Karin M. The wolf in sheep's clothing: the role of interleukin- 6 in immunity, inflammation and cancer. Trends Mol Med. 2008; 14:109-19. https://doi. org/10.1016/j.molmed.2007.12.007
32. Jain S, Harris J, Ware J. Platelets: linking hemostasis and cancer. Arterioscler Thromb Vasc Biol. 2010; 30:2362-67. https://doi.org/10.1161/ATVBAHA.110.207514

33. Shi C, Pamer EG. Monocyte recruitment during infection and inflammation. Nat Rev Immunol. 2011; 11:762-74. https://doi.org/10.1038/nri3070

34. Pollard JW. Tumour-educated macrophages promote tumour progression and metastasis. Nat Rev Cancer. 2004; 4:71-78. https://doi.org/10.1038/nrc1256

35. Low HB, Png CW, Li C, Wang Y, Wong SB, Zhang Y. Monocyte-derived factors including PLA2G7 induced by macrophage-nasopharyngeal carcinoma cell interaction promote tumor cell invasiveness. Oncotarget. 2016; 7:55473-90. https://doi.org/10.18632/oncotarget.10980

36. Chang WA, Hung JY, Tsai YM, Hsu YL, Chiang HH, Chou SH, Huang MS, Kuo PL. Laricitrin suppresses increased benzo(a)pyrene-induced lung tumor-associated monocytederived dendritic cell cancer progression. Oncol Lett. 2016; 11:1783-90. https://doi.org/10.3892/ol.2016.4153

37. Hanahan D, Weinberg RA. Hallmarks of cancer: the next generation. Cell. 2011; 144:646-74. https://doi. org/10.1016/j.cell.2011.02.013

38. Rabinowich H, Cohen R, Bruderman I, Steiner Z, Klajman A. Functional analysis of mononuclear cells infiltrating into tumors: lysis of autologous human tumor cells by cultured infiltrating lymphocytes. Cancer Res. 1987; 47:173-77.

39. Schreiber RD, Old LJ, Smyth MJ. Cancer immunoediting: integrating immunity's roles in cancer suppression and promotion. Science. 2011; 331:1565-70. https://doi. org/10.1126/science. 1203486

40. Sun W, Zhang L, Luo M, Hu G, Mei Q, Liu D, Long G, Hu G. Pretreatment hematologic markers as prognostic factors in patients with nasopharyngeal carcinoma: neutrophillymphocyte ratio and platelet-lymphocyte ratio. Head Neck. 2016 (Suppl 1); 38:E1332-40. https://doi.org/10.1002/ hed. 24224 